\title{
Comparison of three diffusion encoding schemes for cardiac imaging under free breathing conditions.
}

\author{
Kévin Moulin ${ }^{1,2^{*}}$, Alban Chazot $^{3}$, Jérôme Chaptinel ${ }^{4}$, Pierre Croisille ${ }^{1,3}$, Magalie Viallon ${ }^{1,3}$ \\ From 19th Annual SCMR Scientific Sessions \\ Los Angeles, CA, USA. 27-30 January 2016
}

\begin{abstract}
Background
Diffusion cardiac imaging has been challenging due to respiratory and heart motion. Recent developments in cardiac diffusion imaging proposed more robust spin echoes encoding scheme, like Acceleration Motion Correction(AMC)(1), to tackle cardiac motion. In addition, free breathing acquisition with prospective motion correction like slice following technique (2) has been shown to reduce efficiently and significantly the scan time. In this study, the effect of breathing motion on accuracy and precision using AMC, Stjekal-Tanner (Monopolar) and Twice Refocused Spin Echo (TRSE) encoding schemes combined with slice following was evaluated on a moving phantom. In-vivo comparison was achieved in volunteers.
\end{abstract}

\section{Methods}

Monopolar, TRSE and AMC schemes were first evaluated in-vitro: a phantom composed of $2 \%$ agar gel with different sucrose concentrations $(0,5,10,15 \%)$ was translated by a linear motor to reproduce the breathing motion with $4 \mathrm{~cm}$ of amplitude and a frequency of 0.25 $\mathrm{hz}$ (3). Five slices, 6 diffusion directions with b-values of $0,15,30,50,75,100,200,300,400 \mathrm{~s} / \mathrm{mm}^{2}$ and 3 averages were acquired with and without motion on a $3 \mathrm{~T}$ scanner. $\mathrm{TE}=38,54,62 \mathrm{~ms}$ for Monopolar, TRSE and $A M C$ respectively; TR $=5 \mathrm{~s}$. Prospective motion correction was realized using a cross-pair navigator and slice following.

The three same acquisition strategies were compared on 7 volunteers. A 2-min ADC protocol was used: 5 slices, 6 diffusion directions and b-values $0,200 \mathrm{~s} / \mathrm{mm}^{2}$. Five TDs shifted every $10 \mathrm{~ms}$ were acquired to assess cardiac motion by PCAtMIP reconstruction (4). Monopolar, TRSE and AMC were acquired in diastole and AMC in diastole and systole with $\mathrm{TE}=38,54,62 \mathrm{~ms}$ respectively; $\mathrm{TR}=5 \mathrm{~s}$.

An objective artefact quantification was calculated related to diffusion image weighted signal $(S(x, y, i))$ and to $\mathrm{S} 0(\mathrm{x}, \mathrm{y})$, the non-weighted reference image.

\section{Results}

Similar range of errors were found in the moving phantom for the three encoding scheme. The mean error was $3.5 \%$ showing that translational motion like breathing-motion affected lightly the weighting signal (Figure 1 Table a). In-vivo comparison (Figure 2) revealed a high score of artifacts for the Monopolar and TRSE encoding schemes leading to important errors in the ADC measurement: 2.71 and $3.13 * 10^{\wedge}-3 \mathrm{~mm}^{2} / \mathrm{s}$ respectively (Figure1 Table b). Conversely AMC appears robust to cardiac motion with low corresponding values of artefact measurement. Values of ADCs from AMC acquisition are coherent with the literature for both diastolic and systolic phase: 1.94 and $1.44{ }^{*} 10^{\wedge}-3 \mathrm{~mm}^{2} / \mathrm{s}$, respectively.

\section{Conclusions}

Breathing motion compensation like slice following provides motion independent $\mathrm{ADC}$ estimates whatever the diffusion encoding scheme, even for ones with a longer TE (AMC, TRSE). But, cardiac and bulk motions are in opposite very critical for ADC measures, therefore they require an adequate corrected diffusion encoding scheme.

\footnotetext{
${ }^{1}$ CREATIS; CNRS (UMR 5220); INSERM (U1044); University of Lyon, Lyon, France

Full list of author information is available at the end of the article
} 


\begin{tabular}{|c|c|c|c|c|c|c|c|c|c|c|c|}
\hline a) & \multicolumn{4}{|c|}{ Monopolar } & \multicolumn{4}{|c|}{ TRSE } & \multicolumn{3}{|c|}{ AMC } \\
\hline Phantom & $\begin{array}{l}\text { ADC no } \\
\text { motion }\end{array}$ & $\begin{array}{c}\text { ADC } \\
\text { motion }\end{array}$ & \multicolumn{2}{|c|}{ Difference (\%) } & \multicolumn{2}{|c|}{$\begin{array}{l}A D C \text { no } \\
\text { motion }\end{array}$} & $\begin{array}{c}\text { ADC } \\
\text { motion }\end{array}$ & Difference (\%) & $\begin{array}{l}\text { ADC no } \\
\text { motion }\end{array}$ & $\begin{array}{c}\text { ADC } \\
\text { motion }\end{array}$ & Difference (\%) \\
\hline Tube1 & 0,00176 & 0,00182 & \multicolumn{2}{|c|}{3,16138} & \multicolumn{2}{|c|}{0,00173} & 0,00181 & 4,60034 & 0,00175 & 0,00182 & 4,14657 \\
\hline Tube2 & 0,00196 & 0,00202 & \multicolumn{2}{|c|}{2,88449} & \multicolumn{2}{|c|}{0,00206} & 0,00205 & 0,56089 & 0,00204 & 0,00193 & 5,48149 \\
\hline Tube3 & 0,00157 & 0,00167 & \multicolumn{2}{|c|}{5,91916} & \multicolumn{2}{|c|}{0,00155} & 0,00146 & 6,10988 & 0,00158 & 0,00158 & 0,47972 \\
\hline Tube4 & 0,00220 & 0,00224 & \multicolumn{2}{|c|}{1,84636} & \multicolumn{2}{|c|}{0,00225} & 0,00221 & 1,90076 & 0,00224 & 0,00219 & 2,34499 \\
\hline \multirow[t]{2}{*}{ b) } & \multicolumn{3}{|c|}{ Monopolar } & \multicolumn{4}{|c|}{ TRSE } & \multicolumn{4}{|c|}{ AMC } \\
\hline & & & & & & & & \multicolumn{2}{|c|}{ Diastole } & \multicolumn{2}{|c|}{ Systole } \\
\hline Volunteer & $A D C$ & \multicolumn{2}{|c|}{$|\varepsilon|$} & \multicolumn{2}{|c|}{$A D C$} & & $|\varepsilon|$ & $A D C$ & $|\varepsilon|$ & $A D C$ & $|\varepsilon|$ \\
\hline 1 & 0,00182 & \multicolumn{2}{|c|}{0,18986} & \multicolumn{2}{|c|}{0,00199} & & 22890 & 0,00208 & 0,20518 & 0,00185 & 0,16595 \\
\hline 2 & 0,00294 & 0,32 & 099 & 0,0028 & & & 29244 & 0,00189 & 0,20297 & 0,00128 & 0,13998 \\
\hline 3 & 0,00203 & 0,23 & 481 & 0,002 & & & 24186 & 0,00192 & 0,17978 & 0,00142 & 0,14884 \\
\hline 4 & 0,00364 & 0,38 & 487 & 0,003 & & & 43219 & 0,00209 & 0,21721 & 0,00143 & 0,18257 \\
\hline 5 & 0,00200 & 0,14 & 082 & 0,004 & & & 58198 & 0,00171 & 0,21267 & 0,00156 & 0,13419 \\
\hline 6 & 0,00228 & 0,33 & 260 & 0,002 & & & 30337 & 0,00192 & 0,22520 & 0,00121 & 0,12059 \\
\hline 7 & 0,00425 & 0,43 & 913 & 0,004 & & & 44186 & 0,00195 & 0,18380 & 0,00132 & 0,15067 \\
\hline Mean & 0,00271 & 0,29 & 187 & 0,003 & & & 36037 & 0,00194 & 0,20383 & 0,00144 & 0,14897 \\
\hline
\end{tabular}

\section{Authors' details}

'CREATIS; CNRS (UMR 5220); INSERM (U1044); University of Lyon, Lyon,

France. ${ }^{2}$ MRI Department, Siemens Healthcare, Paris, France. ${ }^{3}$ Department of

Radiology/CHU de Saint Etienne, University of Saint Etienne, Saint Etienne,

France. ${ }^{4} \mathrm{ClBM} /$ Radiology Department, University of Lausanne, Lausanne,

Switzerland.

Published: 27 January 2016

\section{References}

1. Nakamura T: ISMRM 2014.

2. Moulin K: ISMRM 2015.

3. Huber ME, et al: J Cardiovasc Magn Reson 2000.

4. Pai VM, et al: Magn Reson Med 2011.

\section{doi:10.1186/1532-429X-18-S1-W16}

Cite this article as: Moulin et al:: Comparison of three diffusion

encoding schemes for cardiac imaging under free breathing

conditions.. Journal of Cardiovascular Magnetic Resonance 2016 18(Suppl

1):W16.

Submit your next manuscript to BioMed Central and take full advantage of:

- Convenient online submission

- Thorough peer review

- No space constraints or color figure charges

- Immediate publication on acceptance

- Inclusion in PubMed, CAS, Scopus and Google Scholar

- Research which is freely available for redistribution 


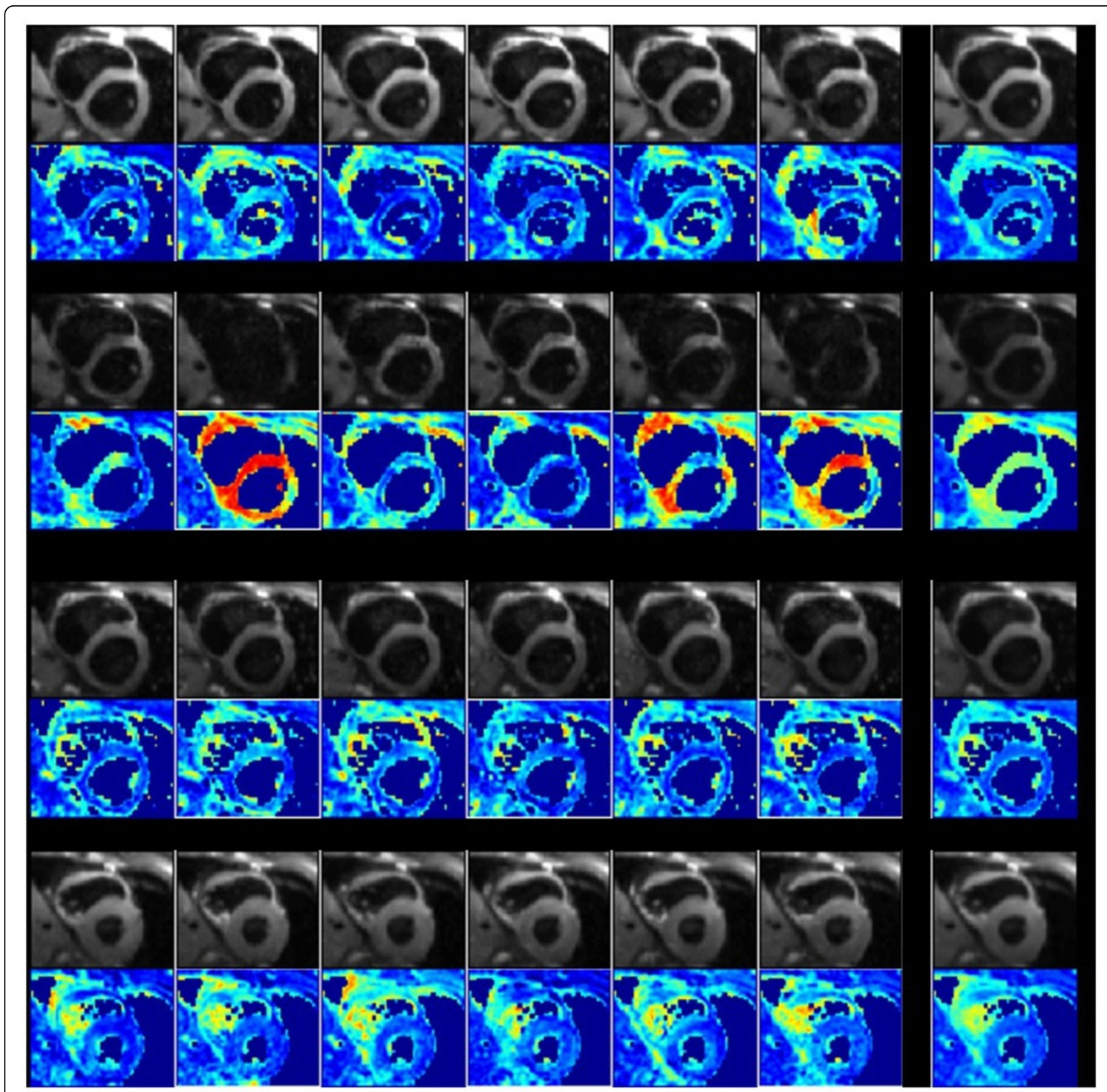

Figure 2 Example of ADC raw images of mid-ventricular short axis slices. In gray scale the Diffusion Weighted Image (DWI) for the 6 directions, b-value $=200 \mathrm{~mm}^{2} / \mathrm{s}$ and the Trace image. In color scale, the corresponding error map,corresponding to the artefact quantification $\varepsilon$. When the DWI signal drop out, artefact quantification reveal higher value. 